\title{
Vermeidbares Leid: Noceboeffekte und die COVID-19-Pandemie
}

\author{
Karin Meissner \\ Fachbereich Integrative Gesundheitsförderung, Hochschule für Angewandte Wissenschaften Coburg, Coburg, \\ Deutschland
}

Die Placeboforschung hat in den letzten Jahrzehnten eindrucksvoll gezeigt, dass Placeboeffekte keine Einbildung sind, sondern messbare physiologische Korrelate haben und bei vielen Beschwerden einen relevanten Teil der Wirkung der "echten" Therapie ausmachen können. Placeboeffekte werden auf positive Erwartungen und Lernerfahrungen zurückgeführt, aber auch die Fürsorge durch andere spielt eine Rolle. Nicht so bekannt ist der "böse Zwilling" des Placeboeffekts - der Noceboeffekt. Ein zentrales Agens des Noceboeffekts ist die Angst.

Ängste spielen auch im aktuellen Kontext der COVID-19-Pandemie eine große Rolle. So berichteten beispielsweise 50\% von 6'500 befragten Personen in Deutschland über Ängste und Belastungen in den ersten Wochen der Pandemie [1]. Die tägliche Beschäftigung mit der Pandemie betrug mehr als 4 Stunden am Tag. Dabei waren nicht eigene Erfahrungen mit dem Coronavirus für das Ausmaß an Angst entscheidend, sondern psychosoziale Faktoren, wie die Akzeptanz negativer Emotionen, Selbstwirksamkeit und soziale Unterstützung. Steigert die COVID-19-Pandemie das Risiko für Noceboeffekte?

Noceboeffekte können bei jeder medizinischen Behandlung eine Rolle spielen. Sie äußern sich z. B. in der Verstärkung von Symptomen oder Nebenwirkungen von Therapien. Allein das Wissen um konkrete Beschwerden als Folge einer Therapie erhöht bereits die Wahrscheinlichkeit, dass diese im Verlauf der Behandlung auftreten. Noceboeffekte schwächen nachweislich die Wirkung auch potenter Schmerzmittel ab. Und das Absetzen einer Schmerzmittelinfusion führt zu einem Wiederanstieg der Schmerzen - allerdings nur bei denjenigen Patienten, die vorab über das Absetzen informiert wurden [2].
Für das Auftreten von Noceboeffekten im klinischen Alltag sind vor allem negative Erwartungen verantwortlich: Diese werden durch einschlägige Vorerfahrungen erlernt und durch Worte und Hinweise induziert. So steigert die gutgemeinte Warnung beim Blutabnehmen "Jetzt tut es gleich weh" den Einstichschmerz. Kurz gesagt kann jede gesundheitsbezogene Information, die Ängste weckt, auch den Noceboeffekt triggern. Dieser ist, wie der Placeboeffekt, keine Einbildung, sondern geht mit messbaren körperlichen Änderungen einher, z. B. der Ausschüttung von Cholezystokinin im Gehirn [3]. Auch Persönlichkeitsmerkmale werden mit Noceboeffekten in Verbindung gebracht: So scheinen ängstliche, belastete Personen mit Somatisierungstendenzen stärker zu Noceboeffekten zu neigen als psychisch stabile Menschen [2].

Noceboeffekte wurden bisher vor allem im Zusammenhang mit medizinischen Aufklärungsgesprächen und Interventionen untersucht. Aber auch Medienberichte können Noceboeffekte nachweislich triggern. So setzten kurz nach Erscheinen von Medienberichten zur Unverträglichkeit von Statinen vor allem ältere Patienten diese Medikamente auch bei langjähriger Einnahme wieder ab [4]. Als eine Gruppe internationaler Experten haben wir vor kurzem Ansätze formuliert, wie Noceboeffekte im klinischen Alltag minimiert werden können [5]. So sollten Behandler und Patienten über die Existenz von Noceboeffekten aufgeklärt werden. Die Patienten werden dadurch befähigt, sich aktiv vor Noceboeffekten zu schützen, z. B. indem sie auf das detaillierte Lesen des Beipackzettels ihres Medikaments oder einschlägiger Blogs im Internet verzichten. Ärzte und Therapeuten wiederum sollten lernen, wie sie ihre Patienten über Nebenwir- 
kungen aufklären, ohne unnötig Ängste zu wecken. Wichtig hierbei ist, nicht nur die potenziell schädigenden Wirkungen, sondern auch die positiven Aspekte der Therapie vor Augen zu führen. Anstelle von Nebenwirkungsraten könnte berichtet werden, welcher Anteil der Patienten die Therapie gut verträgt. Auch Gespräche mit Patienten über mögliche Nebenwirkungen in der Vergangenheit können helfen, Sorgen und Ängste zu zerstreuen. Grundsätzlich schützt eine empathische und wertschätzende Kommunikation mit dem Patienten vor Noceboeffekten.

Was aus der Noceboforschung bekannt ist, könnte gleichermaßen auch für gesundheitsbezogene Ängste gelten, die im Kontext der gegenwärtigen COVID19-Pandemie geweckt werden [6]. So erhöhen Stress und Ängste, wie sie momentan unter COVID-19 zu beobachten sind, mutmaßlich auch das Risiko für Noceboeffekte. Möglicherweise wirken gut bewährte Therapien aufgrund eines verstärkten Noceboeffekts plötzlich weniger, die Akupunkturbehandlung ist schmerzhafter als sonst, oder eine Corona-Impfung ruft aus Angst vor den im Internet diskutierten Nebenwirkungen stärkere Beschwerden hervor, als sie eigentlich müsste, oder wird womöglich gar nicht erst durchgeführt. Gegen negative Erwartungen, Stress und Ängste im Zusammenhang mit COVID-19 könnte wiederum eine optimierte Form der Aufklärung helfen, die nicht nur die Risiken, sondern auch mögliche Schutzfaktoren im Blick hat. Dabei sollten die Schutzfaktoren sich nicht nur auf die AHA+L Regeln beschränken, sondern auch eine Steigerung der psychologischen und körperlichen Widerstandsfähigkeit gegen die Erkrankung durch individuelle Gesundheitsförderung in den Blick nehmen. Die gesundheitsfördernden Effekte von gesunder Ernährung, Bewegung und Entspannung lassen sich beispielsweise bereits nach wenigen Wochen nachweisen - ein Zeitraum, der für die aktuelle Pandemie durchaus von Relevanz ist. Gerade auch für
Personen mit lebensstilassoziierten Vorerkrankungen, welche einen schweren COVID-19-Verlauf begünstigen, könnten Hinweise, was jeder selbst tun kann, um seine Gesundheit zu stärken, hilfreich sein, um starken Ängsten und negativen Erwartungen im Hinblick auf eine mögliche Coronainfektion entgegenzuwirken. Vor starken Ängsten schützen können aber nicht zuletzt auch Institutionen, die gesundheitsrelevante Informationen zur Pandemielage vermitteln. So stellten Amanzio et al. [6] kürzlich öffentliche Aussagen, die das Negative betonen (z. B. "The pandemic alarm will last a long time"; WHO, 15.5.2020), solchen gegenüber, die potenziell beruhigend wirken (z. B. "Proceeding together on research, production and distribution of the vaccine"; Italiens $\mathrm{CO}$ VID-19-Gesundheitsminister an die G7 und EU Staaten, 7.5.2020). Generell plädieren die Autoren für ein ausgewogeneres Verhältnis zwischen negativen und positiven Informationen in der Gesundheitskommunikation, wobei der Schwerpunkt stärker auf den präventiven, diagnostischen und prognostischen Möglichkeiten liegen sollte.

Die COVID-19-Pandemie stellt die medizinische Versorgung zweifellos vor besondere Herausforderungen. Aber gerade jetzt ist auch ein integrativer Behandlungsansatz, der den Patienten in seinem gesamten biopsychosozialen Kontext in den Blick nimmt, besonders wichtig. Ängste vor Behandlungen und ihren Nebenwirkungen könnten gegenwärtig größer sein als gewohnt, der Bedarf an Gesprächen entsprechend höher. Das Wissen aus der Noceboforschung kann helfen, die Wahrnehmung für eine gelungene Kommunikation mit dem Patienten zu schärfen, mit dem Ziel, Ängste und somit auch das Risiko für Noceboeffekte zu minimieren.

\section{Conflict of Interest Statement}

The author has no conflicts of interest to declare.

\section{Literatur}

1 Petzold MB, Bendau A, Plag J, Pyrkosch L, Mascarell Maricic L, Betzler F, et al. Risk, resilience, psychological distress, and anxiety at the beginning of the COVID-19 pandemic in Germany. Brain Behav. 2020 Sep;10(9): e01745.

2 Colloca L, Barsky AJ. Placebo and nocebo effects. N Engl J Med. 2020 Feb;382(6):554-61.

3 Benedetti F, Amanzio M, Vighetti S, Asteggiano $\mathrm{G}$. The biochemical and neuroendo- crine bases of the hyperalgesic nocebo effect. J Neurosci. 2006 Nov;26(46):12014-22.

4 Matthews A, Herrett E, Gasparrini A, Van Staa T, Goldacre B, Smeeth L, et al. Impact of statin related media coverage on use of statins: interrupted time series analysis with UK primary care data. BMJ. 2016 Jun 28; 353:i3283.

5 Evers AW, Colloca L, Blease C, Gaab J, Jensen $\mathrm{KB}$, Atlas LY, et al.; Consortium of Place- bo Experts. What should clinicians tell patients about placebo and nocebo effects? Practical considerations based on expert consensus. Psychother Psychosom. 2021;90(1): 49-56.

6 Amanzio M, Howick J, Bartoli M, Cipriani GE, Kong J. How do nocebo phenomena provide a theoretical framework for the COVID-19 pandemic? Front Psychol. 2020 Oct; 11:589884 